\title{
THE RESEARCH OF CORRELATION OF WATER SURFACE SPECTRAL AND SEDIMENT PARAMETERS
}

\author{
Li Jing ${ }^{1,2^{*}}$, Gong Mingjie ${ }^{1,2}$, Fang Weida ${ }^{1,2}$, Sun Weihong ${ }^{1,2}$ \\ ${ }^{1}$ Jiangsu Province Surveying \& Mapping Engineering Institute, Nan Jing,China-(383497197,9450735, 615984029, \\ 446972930)@qq.com
}

${ }^{2}$ Key Laboratory of Satellite Mapping Technology and Application, National Administration of Surveying, Mapping and Geoinformation, Nan Jing, China -(383497197,9450735, 615984029, 446972930)@qq.com

KEY WORDS: Spectral Reflectance, Sediment, Chroma Indicators, Sensitive Wave Bands

\begin{abstract}
:
In the method of survey underwater topography using remote sensing, and the water surface spectral reflectance $\mathrm{R}$, which remote sensing inversion results were closely related to affects by the water and underwater sediment and other aspects, especially in shallow nearshore coastal waters, different sediment types significantly affected the reflectance changes. Therefore, it was of great significance of improving retrieval accuracy to explore the relation of sediment and water surface spectral reflectance. In this study, in order to explore relationship, we used intertidal sediment sand samples in Sheyang estuary, and in the laboratory measured and calculated the chroma indicators, and the water surface spectral reflectance. We found that water surface spectral reflectance had a high correlation with the chroma indicators; research result stated that the color of the sediment had an very important impact on the water surface spectral, especially in Red-Green chroma $a^{*}$.Also, the research determined the sensitive spectrum bands of the Red-Green chroma $\mathrm{a}^{*}$, which were 636-617nm、716-747nm and 770-792nm.
\end{abstract}

\section{INTRODUCTION}

According to the principle of water body remote sensing, a part of the emitted light of the sun was reflected back by water surface, and a part got into the water after it got to the water surface from the sun. After a complex process of light scattering, light absorption and the reflection of the bottom sediment, the part in the water then left with the water-leaving radiances and received by the sensors. Water-color remote sensing was an application example. We could get the concentration of each component of water through a series of inversion algorithms on the base of the relationship between the light component received by the sensors and the optical properties of the water. But this process was quite intricate in the inshore shallow water, when light could reach the bottom and was reflected(Shunlin Liang, 1988a,and Zhongliang Ping,1982a), because the reflectance of the light was influenced by the bottom sediment (sand, silt or mud)( LyzengeDR.Passive,1978a,

PhiloptWD.Radiative,1987a,William Philpot,Curtiss O Davis, W Paul Bissett,2003a,Fuxing Dang, 2001a, Fuxing Dang, 2008a,andRonghua Ma, 2008a).When all other factors were the same, different bottom sediment often led to different water-leaving radiances and resulted in retrieval error. For instance, on the study of water depth mapping from remote sensing data, the reflectance of water was obviously different in two areas with some water depths ,but different bottom sediment.

So the bottom sediment was one of the main factors that affected the water-leaving radiances. A scholar had brought up a concept called "comprehensive

\footnotetext{
* Author: Li Jing, email:383497197@qq.com; Supported by Marine Science and Technology Innovation Program "Resource Distributions and Tidal Flat Evolutions in Jiangsu Province (HY2017-2) "
} 
impact factors of water depths"(Ying Zhang,1998a). Apparently bottom sediment was one of the most important in those factors(Ying Zhang, 2008a).

Therefore, the spectrum received from the water surface had a close correlation with the bottom sediment, and the research on this correlation was very necessary in order to improve the accuracy of water depth remote sensing. This article would focus on this correlation. Previously, the bottom sediment should be divided into different types. We preliminary proposed two standards: granularity parameters and chrominance index based on their physical and chemical characteristics.

\section{RESEARCH METHODS}

\subsection{Collect And Deal With Soil Samples}

(1)Sediment samples were collected in coastal intertidal zone of Sheyang estuary in Jiangsu Province, and we randomly collected 12 samples of surface sediment.

(2)In a cloudless and good light conditions about 12 am in the noon, the sediment samples were put into a little black basin which was filled with water. When they fully precipitated (sedimentation time preferably more than 24 hours), Water-leaving radiances $\left(\mathrm{L}_{\mathrm{w}}\right)$ of water surface and standard reflection plate was measured using fiber optic spectrometer. Then the spectral reflectance of water surface (R) was calculated.

(3)The correlation between spectral reflectance and chrominance indicators of sediment samples was analysed. The sediment parameters greatly affecting the spectral of water surface were selected, and the corresponding sensitive wave bands were preliminary set using the technology of derivative spectrum.

2.2 Water Surface Spectral Measurement And Deal With

In order to acquire 12 samples of sediment grain size of water surface spectral, we used 12 small bowls, whose diameter was $13 \mathrm{~cm}$, the length was $8 \mathrm{~cm}$, and we paint them brush into black(regarding them as dark, no light reflection ), According to the sample of the serial number one by one into the basin, and then filled with water. Let it precipitation in full(time more than 24), and assumed that the water of suspended particles of light scattering is negligible, In sunny noon 12 o'clock, measured each a small basin of water from water radiant reflection, recorded the spectrometer dark current value (noise) as well as standard reflective plate values, and converted the surface spectral reflectance according to the following formula:

$$
R=\frac{L_{S}-L_{d}}{L_{r}-L_{d}}
$$

$L_{S}$ as each of the sample measured radiation from the

water, $L_{d}$ as the spectrometer dark current value,

$L_{r}$ as standard baffle of the measured values (the reflection of the solar irradiance under $100 \%$ ), after denoising and smoothing the reflection curve, we obtained the result as figure 1 .

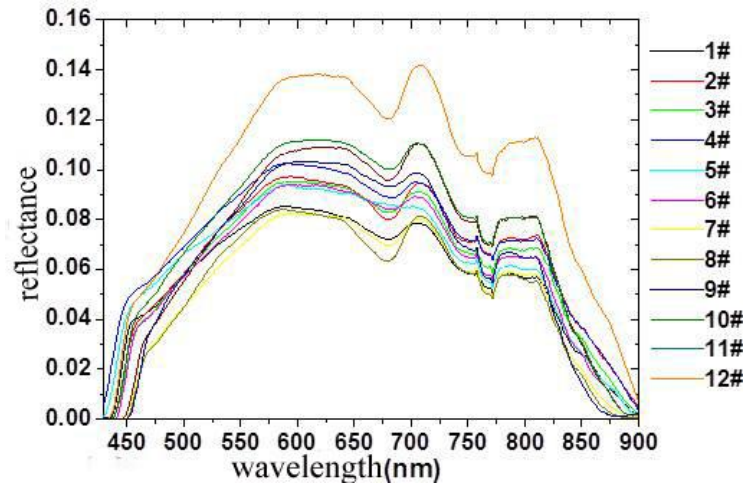

Figure 1. the samples of water surface spectral reflectance

From figure 1, we knew that spectral reflectance curve in the range of $430 \mathrm{~nm}$ and $920 \mathrm{~nm}$.there were 3 obvious reflection peaks, and the first reflection peak was between $570 \mathrm{~nm}$ and $650 \mathrm{~nm}$, the second reflection peak was between $680 \mathrm{~nm}$ and $720 \mathrm{~nm}$, and the third was $780 \mathrm{~nm}-820 \mathrm{~nm}$.the third reflection peak height was significantly lower than the first, the second reflection peak. 


\section{THE CORRELATION ANALYSIS OF WATER SURFACE SPECTRAL REFLECTANCE AND CHROMINANCE INDICATORS}

Based on predecessors' study of spectrum and chromaticity, we found that they had a close relationship between spectrum and chromatic index, and changes of chrominance had a profound impact on spectrum (BARRANCO F T,1989a,GIOSAN L,2002a,and Kunshan Wang,2006a.).so, we made a preliminary study on the relationship between the index and water surface spectrum.

3.1 The Definition And Measurement of Sediment Chrominance Indicators

In general, what influenced the sediment color may be sediment composition and chemical properties that were sensitive to chrominance, including the chemical composition of organic matter, carbonate content, specific chemical composition (such as oxidizing and reducing substances) (Wang Kunshan,2007a). Nearshore submarine sediment affected by terrigenous material, especially the influence of the transport of sediment of large rivers, the color of the bottom sediment was roughly same with suspended sediment in water color. In some weaker ocean dynamics area, due to marine organisms and marine sedimentation, organic carbon content influenced the color change. In the quantitative description, color mainly used chrominance indicators to describe, including luminance and chroma, and in CIELAB color table system was described by three parameters $L^{*}, a^{*}, b^{*}$. L*shows brightness, its value changes between black (0) and white (100), and the deeper of the color of the sediment, the smaller the value, conversely, the greater. $\mathrm{a}^{*}$ is on behalf of the Red-Green color, the value changes between green (60) and red (60), b* means Yellow-Blue color, the value of the change is between blue (60) and yellow (60).According to the predecessors' research, chroma $\mathrm{Ca}^{*} \mathrm{~b}^{*}\left(\mathrm{Ca}^{*} \mathrm{~b} *=\left[\left(\mathrm{a}^{*}\right) 2\right.\right.$ $\left.\left.+\left(b^{*}\right) 2\right]^{1 / 2}\right)$ derived by $a^{*}, b^{*}$ under the influence of $a^{*}, b^{*}$ work together, is able to reflect the general characteristics of sediment chroma change (Lijun Zhu,2000a). In order to quantitatively describe the relationship between the sediment color and the water surface spectral reflectance, we used Colourflex chroma meter to measure the 12 chroma indexes of experimental samples, and according to the measured values calculated the $\mathrm{Ca}^{*} \mathrm{~b} *$. the results listed in table 1.

\begin{tabular}{|c|c|c|c|c|}
\hline ID & $\mathrm{L}$ & $\mathrm{a}^{*}$ & $\mathrm{~b}^{*}$ & $\mathrm{Ca} \mathrm{b}^{*}$ \\
\hline 1 & 46.26 & 4.12 & 15.75 & 16.28 \\
\hline 2 & 46.07 & 4.94 & 17.38 & 18.07 \\
\hline 3 & 50.12 & 4.78 & 16.36 & 17.04 \\
\hline 4 & 42.73 & 4.83 & 16.64 & 17.33 \\
\hline 5 & 53.2 & 4.3 & 15.86 & 16.43 \\
\hline 6 & 38.1 & 4.68 & 16.43 & 17.08 \\
\hline 7 & 36.55 & 4.39 & 15.16 & 15.78 \\
\hline 8 & 40 & 4.48 & 15.57 & 16.20 \\
\hline 9 & 48.5 & 5.47 & 17.57 & 18.40 \\
\hline 10 & 45.73 & 5.28 & 17.15 & 17.94 \\
\hline 11 & 39.27 & 5.33 & 16.26 & 28.90 \\
\hline 12 & 43.68 & 4.44 & 15.97 & 20.21 \\
\hline
\end{tabular}

Table 1. Chromaticity index of experimental samples

\subsection{The Correlation Of Water Surface Spectral \\ Reflectance And Sediment Chrominance} Indicators Analysis

To explore the relationship between reflectance and chromaticity index, we calculated the correlation of reflectance and index of various degrees (figure 2). In addition, due to the $\mathrm{L}, \mathrm{a}^{*}$ and $\mathrm{b}^{*}$ values range were different, we stretched all these three indicators linear up to between 0 and 1 , and the brightness $\mathrm{L}$ shall be carried out in accordance with the formula of $Y=\frac{1}{100} X$ linear stretch, and Red-Green chroma $\mathrm{a}^{*}$ and Yellow-Blue chroma $\mathrm{b}^{*}$ sticked to $Y=\frac{1}{120} X+0.5$ , and $\mathrm{Ca}^{*} \mathrm{~b}^{*}$ would be got according to corresponding formula. the result of correlation analysis with reflectance and results are shown in figure 3 . 


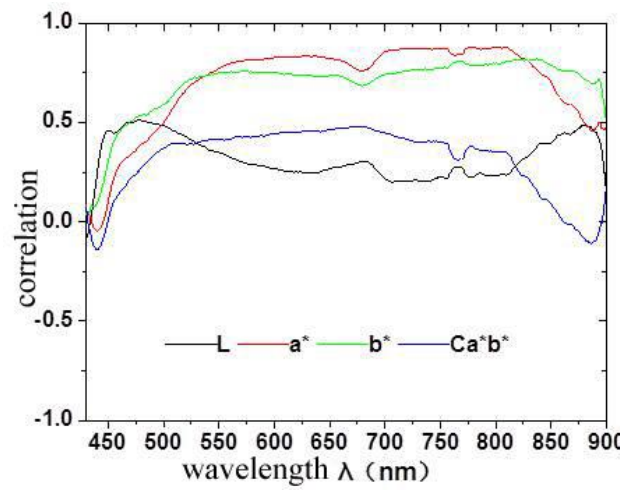

Figure 2. reflectance and various degrees of correlation

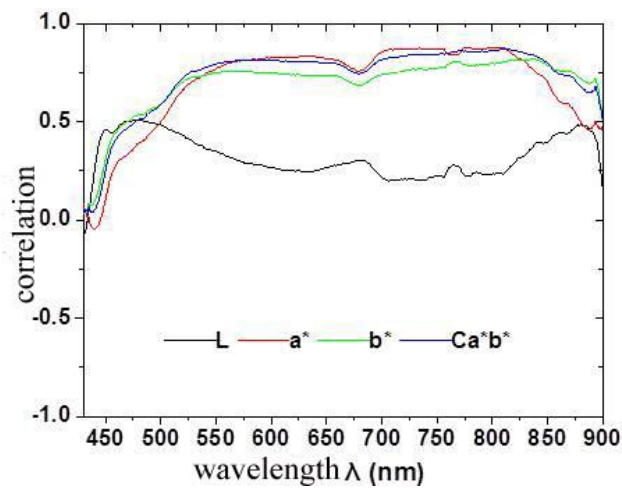

Figure 3. reflectance and the correlation of linear

stretch

Seen from the curve of the figure 2 , the reflectance and the samples of color index showed the following characteristics:

(1) the correlation of reflectance and $a^{*}$ was higher than others between $539 \mathrm{~nm}$ and $827 \mathrm{~nm}$, and the average value was 0.8 or above.

(2) the correlation of reflectance and $b^{*}$ was high too from $539 \mathrm{~nm}$ to $827 \mathrm{~nm}$,but was lower than a*. before $530 \mathrm{~nm}$ and after $827 \mathrm{~nm}$,the correlation was higher than $\mathrm{a}^{*}$.

(3) L and $\mathrm{Ca}^{*} \mathrm{~b}^{*}$ showed low correlation with reflectance within of the whole band. from $517 \mathrm{~nm}$ to $835 \mathrm{~nm}$, the maximum correlation value was not more than 0.5 between reflectance and $\mathrm{L}$. however, the correlation value between chroma $\mathrm{Ca}^{*} \mathrm{~b} *$ and $\mathrm{L}$ was not more than 0.45 .

Seen from the curve of the figure 3 , the correlation between reflectance and chrominance indicators after linear stretch showed the characteristics as follows:

(1)the correlation had the same regulation as figure 2 between $\mathrm{a}^{*}, \mathrm{~b}$ *as well as $\mathrm{L}$ and reflectance.

(2)the correlation had the same regulation as $a^{*}, b^{*}$ between reflectance and after linear stretch of $\mathrm{Ca}^{*} \mathrm{~b} *$. these results showed that the correlation of water surface reflectance and the bottom sediment chroma index was higher, and more stable, especially the correlation of the reflectance and $a^{*}, b^{*}$ had high value areas within a certain range of the wavelength, so we could think that what the sediment had effect on the water surface spectral was the bottom sediment color.

\subsection{Red-Green Chroma A* Spectrum Characteristic Analysis}

According to the above study, we found that the correlation of the Red-Green chroma $a^{*}$, Yellow-Blue chroma $\mathrm{a}^{*}$ and the water spectral reflectance was very stable. And because previous studies have proved that $a^{*}$ had a high correlation with $b^{*}$,so we chose one of them as sediment color parameters to research the relation of sediment color and the spectral. From the last discussion, we knew that the correlation between $\mathrm{a}^{*}$ and the reflectance was higher in $539-827 \mathrm{~nm}$, relatively speaking.so we chose Red-Green chroma a*of chrominance indicators as sediment color parameters, to study the relationship with surface spectrum.

From figure 2 , we knew that $\mathrm{a}^{*}$ showed a relatively high correlation in three spectral bands interval and the concrete details showed in figure 4 .

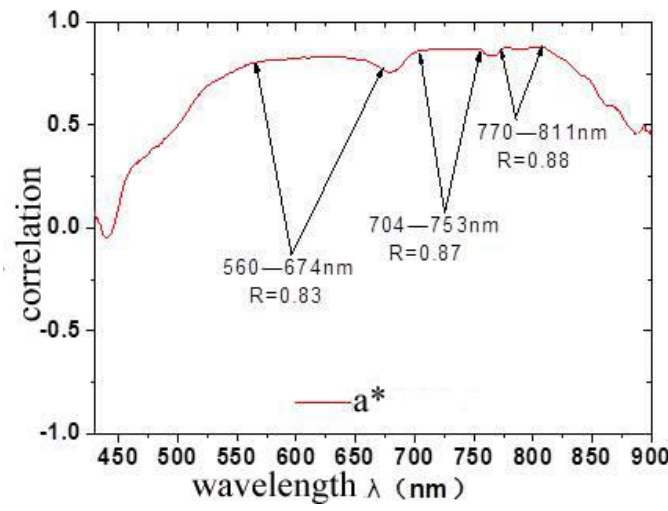

Figure 4. $a^{*}$ with the correlation of reflectance

From figure 4, we can see that there were three intervals where $a^{*}$ had a relative higher correlation with reflectance, the first interval was $560-674 \mathrm{~nm}$, the 
maximum correlation was 0.85 , with an average of 0.83 ; the second was $704-753 \mathrm{~nm}$, the maximum correlation was 0.9 , with an average of 0.87 ; the third interval was $770-811 \mathrm{~nm}$, the correlation maximum was 0.91, with an average of 0.88.these three wave spectral bands may be the sensitive bands of $\mathrm{a}^{*}$.

Differential spectrum was thought to discern the subtle differences of spectral that its values reflect the changes of the spectral reflectance in a certain range(Yun Zhu2007a,Rongqiang Gao 2004a Luda Zhang2005a). changes of series of sample spectrum curve of the first order differential within a certain range, can reflect the changes of the spectrum when the samples were different. if the first order differential changes were well-distributed, the spectral reflectance must have a high correlation with the samples, but this not always showed samples at these spectrum range were very sensitive.

If the first order differential changes were obvious along with the different samples in a certain range, even though the correlation values were not the maximum between the reflectance and the samples in these wave bands, we could also think these bands were sensitive to wave bands for samples.

In order to ensure the sensitive wave bans of the Red-Green chroma $\mathrm{a}^{*}$ in above three intervals, we did the first order differential for spectral reflectance and the three scopes of first order differential of partial enlargement were below.

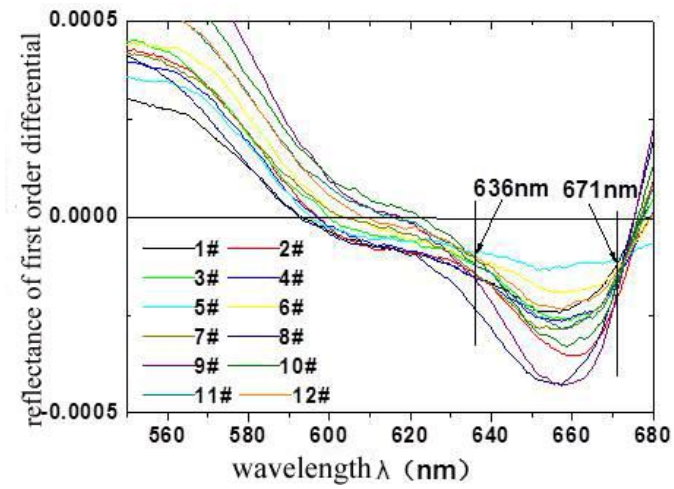

Figure 5. reflectance of first order differential amplifier in $560-674 \mathrm{~nm}$

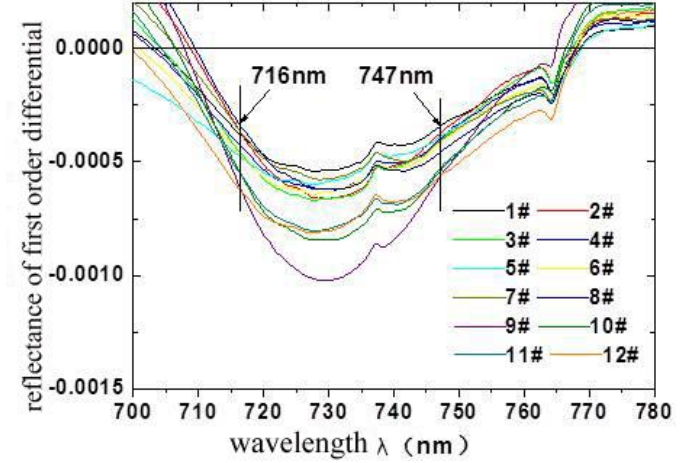

Figure 6. reflectance of first order differential amplifier in 704-753nm

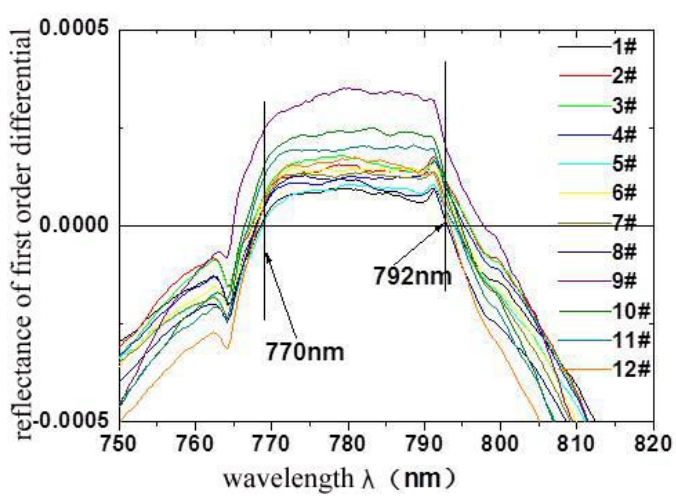

Figure 7. reflectance of first order differential amplifier in770-811nm

From figure 5 and table 1,we could know that in $636-671 \mathrm{~nm}$, the changes of reflectance of the first order differential were obvious, along with the variations of Red-Green chroma a*, according to the above analysis, we chose the $636-671 \mathrm{~nm}$ as spectral sensitive wave bands. Also, from fig6 and fig7,716-747nm and 770-792nm were sensitive bands of $\mathrm{a}^{*}$.In these bands ranges, the correlation between average spectral reflectance and $\mathrm{a}^{*}$ reached $0.81,0.88,0.88$, respectively. These scatter diagrams were below.

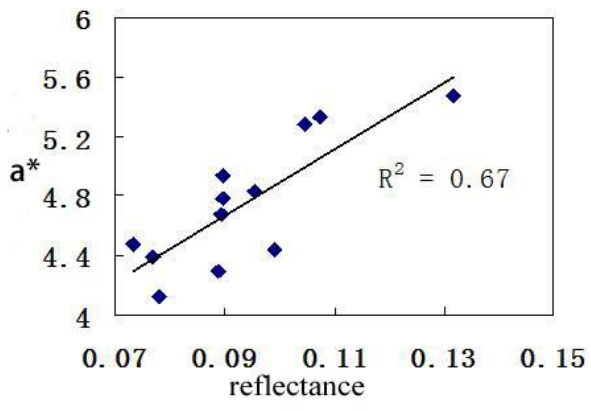

Fig 8 the scatter diagrams between $\mathrm{a}^{*}$ and the reflectance in $636-671 \mathrm{~nm}$ 


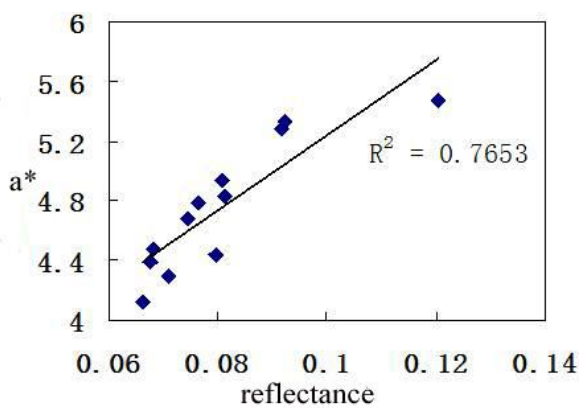

Fig9 the scatter diagrams between $\mathrm{a}^{*}$ and the reflectance in $716-747 \mathrm{~nm}$

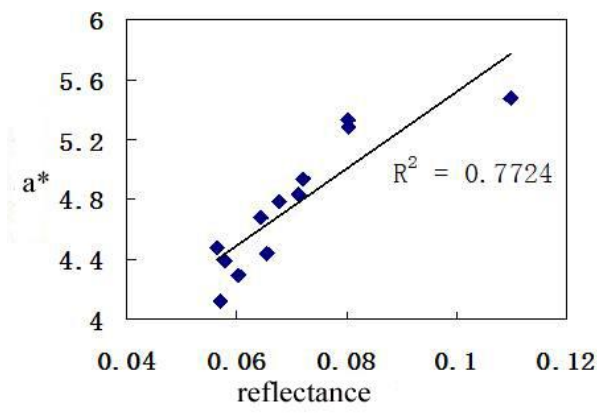

Fig 10 the scatter diagrams between $\mathrm{a}^{*}$ and the reflectance in $770-792 \mathrm{~nm}$

\section{CONCLUTION}

In order to research the relation of water surface spectral and underwater sediment, this paper explored the correlation between water surface spectral reflectance and the bottom color parameters (color index), and the results showed that:

(1) Reflectance had the higher correlation with Red-Green chroma $\mathrm{a}^{*}$ and Yellow-Blue color $\mathrm{b}^{*}$ of the sediment chromaticity index between $539 \mathrm{~nm}$ and $827 \mathrm{~nm}$, with an average of more than 0.8 . so we thought that the influence of sediment on the water surface spectral mainly was the chromaticity index of the sediment.

(2) In the color index, the correlation of spectrum reflectance and Red-Green chroma $\mathrm{a} *$ from $539 \mathrm{~nm}$ to $827 \mathrm{~nm}$ were higher than other some parameters; Within the scope of the correlation, there were three high values area. the first interval was $560-674 \mathrm{~nm}$, the maximum correlation was 0.85 , with an average of 0.83; The second was $704-753 \mathrm{~nm}$, the maximum correlation was 0.9 , with an average of 0.87 ; The third interval was $770-811 \mathrm{~nm}$, the correlation maximum was 0.91 , with an average of 0.88 .
(3) Because Red-Green chroma a* and Yellow-Blue chroma $b^{*}$ were highly correlated, we took $\mathrm{a}^{*}$ for main color parameters of sediment as the research object. In order to determine the sensitive spectral band which was closely related to $\mathrm{a}^{*}$, we calculated spectral reflectance for the first order differential, and ultimately determined $636-617 \mathrm{~nm}, 716-747 \mathrm{~nm}$, and 770-792nm as sensitive spectral bands.

\section{ACKNOWLEDGEMENTS}

This paper was supported by Marine Science and Technology Innovation Program "Resource Distributions and Tidal Flat Evolutions in Jiangsu Province (HY2017-2)".

\section{REFERENCES}

BARRANCO F T, BALSAM W L, DEATON B C,1989a. Quantative reassessment of brick red lutites: Evidence from reflectance spectropho-tometry.Marine Geology, 89,pp.299-314.

Fuxing Dang, Qian Ding,2001a. A study of shallow water depth extraction using Landsat imagery.Remote Sensing for Land \& Resources, 4(1),pp.53-58.

Fuxing Dang, Qian Ding,2008a.A technique for extracting water depth information from multispectral scanner datain the South China Sea. Marine Sciences Bulletin, 22(3),pp. 55-60.

GIOSAN L, FLOOD R D, ALLER R C,2002a. Pale oceanographic significance of sediment color on western North Atlantic drifts:I Origin of color.Marine Geology, 189,pp.25-41.

Kunshan Wang, Xuefa Shi, Guoqing Wang,2006a. A preliminary study on the sediment color reflectance in the Southern Yellow Sea sheft area. Advances in marine science, 24(1),pp.30-38.

Kunshan Wang, Xuefa Shi,2007a. Analysis of affecting factors for reflectance spectra of sediments from the central shelf area of the Southern Yellow Sea. Advances in marine science, 25(1),pp.46-53.

Lijun Zhu, Yuming You,2000a. Remote sensing analysis on coastal process and characteristics of suspensed sediment in Suizhong coast area of Liaodong Bay. Ocean Engneering, 18 (1),pp.65-69.

Luda Zhang, Tao Wang, Liming Yang,2005a. An applied study on fourier transform near-infrared whole spectroscopy regression analysis.Spectroscopy and Spectral Analysis,25(12),pp.1959-1962.

LyzengeDR.Passive,1978a. remote sensing techniques for mapping water depth and bottom features.Applied Optics,17(3),pp.379-383. 
PhiloptWD.Radiative,1987a.transfer in stratified water: A single scattering approximation for irradiance . Applied Optics,26 (19),pp.4123-4132.

Ronghua Ma, Hongtao Duan, Shouxuan Zhang, 2008a. Contribution of vegetation bottom to remote sensing reflectance in Taihu lack, China. Journal of Remote Sensing,12(3),pp.483-489.

Rongqiang Gao, Shifu Fan, Yanlu Yan, 2004a Preprocessing of Near Infrared Spectroscopic data.Spectroscopy and Spectral Analysis, 24(12),pp.1653-1655.

Shunlin Liang, Bingxian Chen,1988a.A study on remote sensing perspective depth into water in visible band. Donghai Marine Science, 6(2),pp.28-36.

William Philpot,Curtiss O Davis, W Paul Bissett,2003a.Bottom Characterization from Hyperspectral Image Data. Oceanography, 17(2),pp.76-85.

Ying Zhang,1998a. Study of fathoming method by RS technology. Journal of hohai University, 26(6),pp.68-72.

Ying Zhang, Dong Zhang, Yianjiao Wang, 2008a. Study of remoting sensing-based bathymetric method in sand-containing water bodies. Acta Oceanologic Sinca, 30(1),pp.51-58.

Yun Zhu, Ye Chen, Qiang Shu,2007a. Chroma Index record of core XH1 at northern Jiangsu Basin and the climate since Mid-Pleistocene. Marine geology \& Quaternary Geology, 27(2),pp.23-37.

Zhongliang Ping,1982a.Mathematics model for visible remote sensing of water depth. Oceanologia ET Limnologia Sinica, 13(3),pp.225-230. 